\title{
Respuesta en poscosecha de frutos de agraz (Vaccinium meridionale Swartz) a almacenamiento en dos tipos de plástico
}

\section{Postharvest response of blueberry fruits (Vaccinium meridionale Swartz) to storage in two types of plastic}

Fernando Javier Peña-Baracaldo ; Hans Nicolás Chaparro²; Helber M. Orjuela-Matta ${ }^{3}$; Gladys Romero-Guerrero ${ }^{4}$

${ }^{1}$ Ing. Agronómo, M.Sc. Universidad de Ciencias Aplicadas y Ambientales, U.D.C.A, Facultad de Ciencias Ambientales e Ingenierías, Producción Agrícola Sostenible. Bogotá, D.C., Colombia; e-mail: fepena@udca.edu.co; Dhttps://orcid.org/0000-0001-7809-9686

${ }^{2}$ Ing. Agrónomo, M.Sc. Universidad de Ciencias Aplicadas y Ambientales, U.D.C.A, Facultad de Ciencias Ambientales e Ingenierías, Producción Agrícola Sostenible. Bogotá, D.C., Colombia; e-mail: hchaparro@udca.edu.co; Dhttps://orcid.org/0000-0002-4355-8050

${ }^{3}$ Ing. Agrícola, M.Sc. Universidad de Ciencias Aplicadas y Ambientales, U.D.C.A, Facultad de Ciencias Ambientales e Ingenierías, Producción Agrícola Sostenible. Bogotá; e-mail: horjuela@udca.edu.co; Dhttps://orcid.org/0000-0002-9116-3096

${ }^{4}$ Bióloga, Ph D., Universidad de Ciencias Aplicadas y Ambientales, U.D.C.A, Facultad de Ciencias Ambientales e Ingenierías, Producción Agrícola Sostenible. Bogotá; e-mail: glromero@udca.edu.co; Dhttps://orcid.org/0000-0001-9394-5174

Cómo citar: Peña-Baracaldo, F.J.; Chaparro, H.N.; Orjuela-Matta, H.M.; Romero-Guerrero; G. 2020. Respuesta en poscosecha de frutos de agraz (Vaccinium meridionale Swartz) a almacenamiento en dos tipos de plástico. Rev. U.D.C.A Act. \& Div. Cient. 23(2):e1730. http://doi.org/10.31910/rudca.v23.n2.2020.1730

Artículo de acceso abierto publicado por Revista U.D.C.A Actualidad \& Divulgación Científica, bajo una licencia Creative Commons CC BY-NC 4.0

Publicación oficial de la Universidad de Ciencias Aplicadas y Ambientales U.D.C.A, Institución de Educación Superior Acreditada de Alta Calidad por el Ministerio de Educación Nacional.

Recibido: Enero 17 de 2020 Aceptado: Octubre 14 de 2020 Editado por: Ingeborg Zenner de Polanía

\section{RESUMEN}

La planta de agraz (Vaccinium meridionale Swartz) es una especie nativa de arándano de Colombia y su fruto es una baya globosa con propiedades nutraceúticas, debido a su excelente fuente de antioxidantes, utilizado como ingrediente para la preparación de alimentos. El objetivo del estudio fue evaluar las propiedades físico químicas del fruto, almacenado durante 12 días, a $2^{\circ} \mathrm{C}$ y $90 \%$ de HR. Se evaluaron frutos en dos estados de madurez: rompimiento de color, estado inmaduro (asociado al color rojo) y completamente pigmentado ( $100 \%$ pigmentación morado), los frutos fueron puestos en bolsas hechas de polietileno biorientado y polipropileno monoorientado. Los resultados muestran que los frutos inmaduros preservaron los sólidos solubles totales, cuando se mantuvieron en polipropileno monoorientado, mientras los sólidos solubles totales, se incrementaron significativamente en los frutos maduros almacenados en polipropileno monorientado, afectando el metabolismo del fruto y las reservas de carbohidratos. $\mathrm{El}$ pH disminuyó en todos los tratamientos; entre los 6 y 12DDT, coincidiendo, a su vez, con el aumento de la acidez total titulable durante el mismo periodo.

Palabras clave: Almacenamiento refrigerado; Acidez total titulable; Agraz empaque; Sólidos solubles totales. 


\section{ABSTRACT}

'Agraz' (Vaccinium meridionale Swartz) is a native blueberry from Colombia, being the fruit a globose berry, with nutraceutic quality due to an excellent source of antioxidants and is used as an ingredient for food preparation. The objective of this study was to evaluate the physical-chemical properties of fruits stored during 12 days, at a temperature of $2^{\circ} \mathrm{C}$ and a relative humidity of $90 \%$. Fruits with two stages of ripeness, color break, an immature state (associated with red color), and fully pigmented (100\% purple pigmentation) were stored, packaged in two different plastics recipients, one bioriented polyethylene and the other mono-oriented polypropylene. The results indicate that immature fruits demonstrated a prolonged preservation of the total soluble solids, when combined with the mono-oriented polypropylene packaging, otherwise TSS were significantly increased in mature fruits, packaged in mono-oriented polypropylene, affecting fruit metabolism and carbohydrate reserve. The $\mathrm{pH}$ dicreased in all treatments, between 6 to 12 days after treatment, coinciding in turn, with the increase in total titratable acidity during the same period.

Keywords: Blueberry packaging; Refrigerated storage; Titratable total acidity; Total soluble solids.

\section{INTRODUCCIÓN}

El agraz (Vaccinium meridionale Swartz) es una especie que crece en condiciones silvestres en la zona Alto Andina, entre los $2.300 \mathrm{y}$ .3500 m s.n.m., siendo una planta de páramo (Patiño \& Ligarreto,
2006). V. meridionale, también se encuentra en la zona norte de los Andes, desde el norte de Ecuador hasta los límites con Venezuela (Briceño \& Morillo, 2002), siendo destacada para Colombia, como especie promisoria frutal de clima frio (Ligarreto Moreno et al. 2010).

La importancia económica del agraz, se centra en las propiedades físico químicas del fruto, por presentar compuestos polifenólicos y antocianinas (Alzate-Arbeláez et al. 2019; Diaz-Uribe et al. 2019), en altas concentraciones, comparado con otras especies de Vacinium de diferente origen (González et al. 2017). Es posible diferenciar agraz de otras especies, dentro de su género, por su único patrón de antocianinas (Garzón et al. 2010), excelente fuente de antioxidante y colorante natural e ingrediente de alimentos funcionales. El fruto (Figura 1) es una baya globosa de, aproximadamente, $0,5 \mathrm{~cm}$ de diámetro ecuatorial, que varía de un color verde en estado inmaduro, pasando por color rojo, llegando a morado, en estado maduro (Buitrago Guacaneme et al. 2015); esto último indica el índice de cosecha, tiempo apropiado para su consumo (Medina Cano et al. 2019).

La maduración fisiológica del fruto, se suele iniciar antes de que termine el crecimiento e, incluye, diferentes procesos, como la madurez del embrión en la semilla, que no está directamente relacionado con la madurez de consumo del fruto (Wills \& Golding, 2016). Para el caso de agraz, se han determinado 5 etapas de maduración del fruto, clasificados de acuerdo con el color de la epidermis, con variaciones fisicoquímicas en cada uno de los estadíos de desarrollo (Buitrago Guacaneme et al. 2015). Estos cambios en características, como sólidos solubles totales y acidez total titulable,

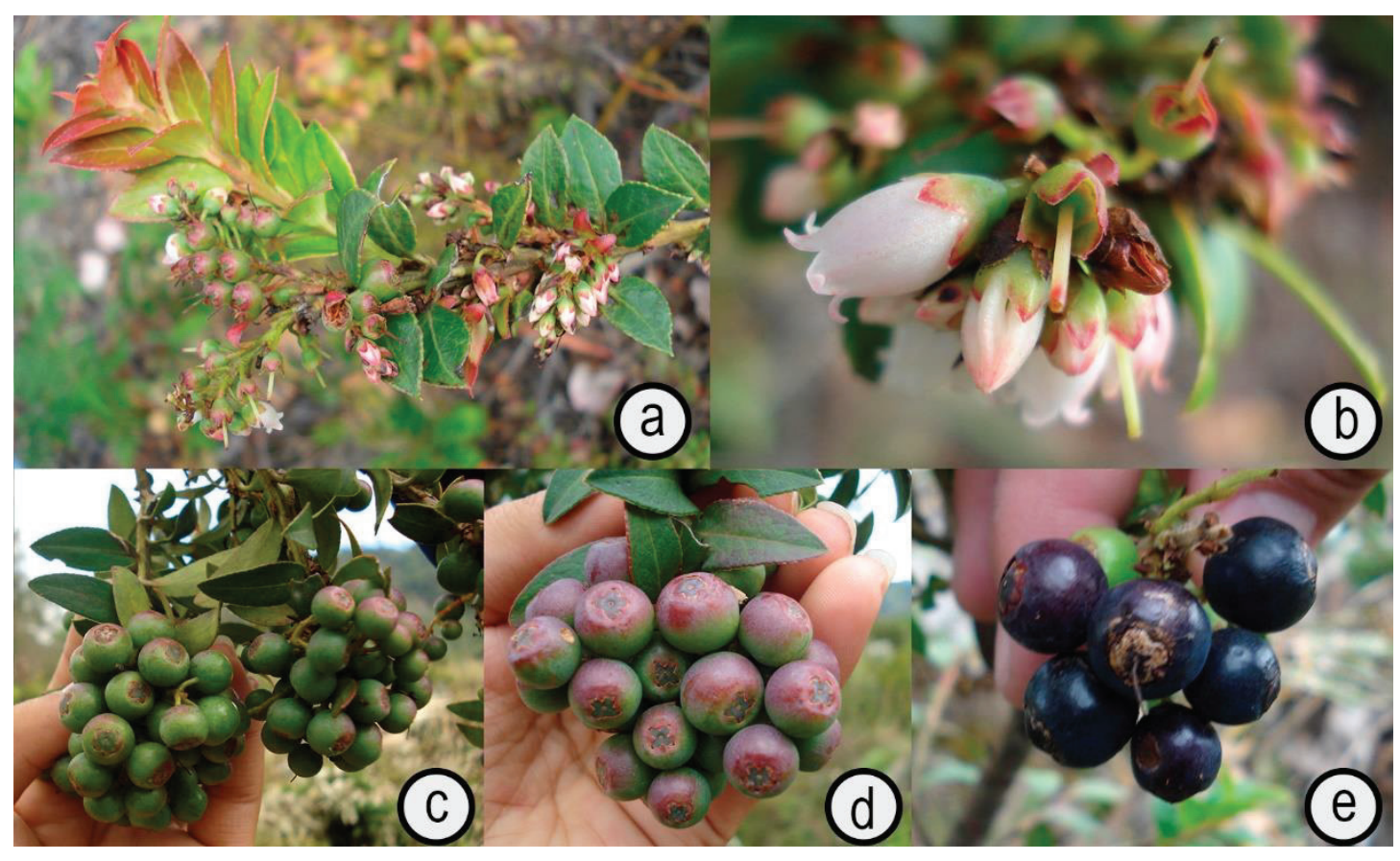

Figura 1. Etapas fenológicas en la producción de agraz. a. Racimo floral; b. Flor de agraz; c. Fruto de agraz inmaduro color verde; d. Fruto de agraz verde rojizo; e. Fruto de agraz en madurez de consumo. 
son identificables en las diferentes etapas de maduración; también, se presenta degradación de ácidos orgánicos durante la respiración del fruto, siendo varios, de estos elementos, componentes esenciales en el ciclo respiratorio de los ácidos tricarboxílicos, junto con una disminución de la acidez durante la maduración de los frutos (Kays et al. 2004). Asimismo, la velocidad de transpiración determina la pérdida de agua del tejido vegetal, siendo influenciada por las características morfológicas o anatómicas, la relación entre superficie y volumen, los daños superficiales y el estado de madurez o también por la temperatura, la humedad relativa, la velocidad y movimiento de aire y la presión atmosférica. En los últimos años, se ha evaluado la vida poscosecha de agraz para temperaturas que oscilan entre 1 y $2^{\circ} \mathrm{C}$, manteniendo un efecto aceptable en las características, que definen la calidad de la fruta (Ávila Rodríguez et al. 2007).

Una de las maneras de controlar el cambio en las características internas y externas de los frutos es el uso de empaques diseñados para modificar la tasa de respiración y transpiración de los frutos (Mangaraj et al. 2009). En frutos, como arándano V. corymbosum, se han empleado empaques de poliactida para prolongar la vida poscosecha de este fruto (Almenar et al. 2008). También, se han evaluado materiales, como polietileno de baja densidad (LDPE), policarbonato, tereftalato de polietileno (PET) y polipropileno PP, para determinar su capacidad de absorción de sabor y permeabilidad al oxígeno (Van Willige et al. 2002). Por otro lado, el uso de polipropileno biorientado (BOPP) ha sido exitoso para el almacenamiento de guayaba, un frutal tropical (Vijayanand et al. 2000); este polímero ha mostrado diversos resultados en cuanto a control de madurez del fruto de litchi, cuando es utilizado en conjunto con atmósferas modificadas (Somboonkaew \& Terry, 2010). Uno de los polímeros más utilizados en evaluaciones poscosecha es el polipropileno monoorientado, demostrando alta eficiencia para prolongar la vida poscosecha de los frutos, frente a los resultados alcanzados con polietileno (Karacay \& Ayhan, 2010).
En razón a las condiciones agroecológicas de Colombia, existe la posibilidad de ofrecer frutos continuamente; sin embargo, es primordial conocer las características físico químicas presentes del producto durante el almacenamiento, con el fin de preservarlas, mediante el uso de diferentes tecnologías poscosecha.

El objetivo del estudio fue evaluar los cambios de las características físico químicas de fruto de agraz durante poscosecha, considerando dos estados de madurez y tipos de empaques comerciales, en almacenamiento en frío.

\section{MATERIALES Y MÉTODOS}

El material vegetal de agraz (Figura 1), se recolectó en campo en la vereda Torres, localizada en el municipio de Ráquira, departamento de Boyacá, Colombia, en las coordenadas Latitud 5³2’26” N, Longitud $73^{\circ} 38^{\prime} 14^{\prime \prime}$ O, a una altitud de $2.635 \mathrm{~m}$ s.n.m. La fruta, se clasificó por color y tamaño, mediante muestreo al azar, de acuerdo con parámetros establecidos sobre el área de influencia, para dos estados de madurez, rojo (inmaduro) y morado (maduro) (Figura 2), según Buitrago Guacaneme et al. (2015). El establecimiento del ensayo y la toma de datos experimentales, se realizaron en el laboratorio de Fitotecnia de la Universidad de Ciencias Aplicadas y Ambientales U.D.C.A. Para los análisis, se tomaron muestras con un peso de $150 \mathrm{~g}$ para cada unidad experimental, con cuatro repeticiones. Posteriormente, se procedió al almacenamiento en dos tipos de empaque, manteniendo las muestras de los productos, a una temperatura $2 \pm 2{ }^{\circ} \mathrm{C}$ y humedad relativa del $90 \pm 5 \%$.

Los cambios de maduración en las dos etapas de maduración de frutos de agraz, se evaluaron a los 0, 3, 6, 9 y 12 días después iniciar los tratamientos (DDT). Se empleó un diseño completo al azar con arreglo de tratamientos factorial. Los factores fueron: dos estados de madurez, de acuerdo con la pigmentación del fruto: 1)

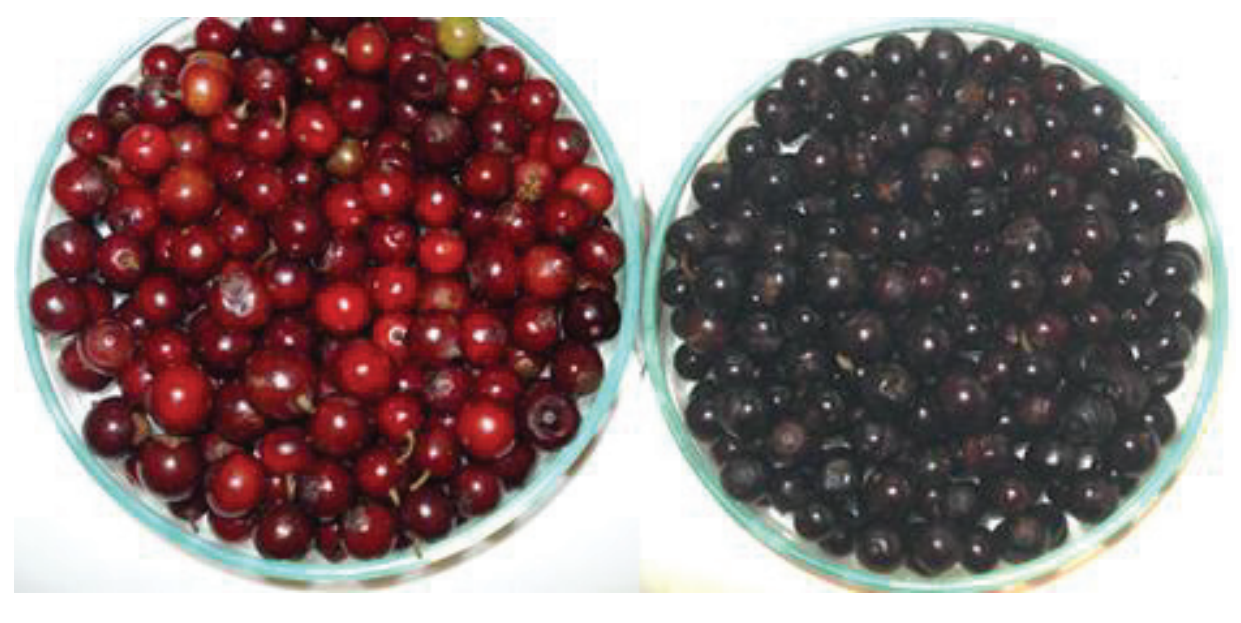

Figura 2. Estados de madurez agraz, izquierda color rojo (inmaduro), derecha color morado (maduro). 
rojo (inmaduro); 2) morado (maduro) y dos tipos de empaque: 1) polietileno biorientado (BOPP, E1) y 2) polipropileno monorientado calibre 1 (MOPP, E2). La combinación de los factores generó cuatro tratamientos. Los tratamientos tuvieron la siguiente nomenclatura: T1 = morado $\times$ BOPP; T2=morado $\mathrm{x}$ MOPP; T3=rojo $\times$ BOPP $y$ T4= rojo $\mathrm{x}$ MOPP.

\section{Variables de respuesta}

Sólidos solubles totales (SST): Fue estimada, mediante el uso de un refractómetro manual, modelo RF15, para medición con escala de 0 a $32 \%$, expresado en ${ }^{\circ}$ Brix. El $\mathrm{pH}$ fue determinado en jugo obtenido a partir de $30 \mathrm{~g}$ de fruta de agraz macerada en $5 \mathrm{~mL}$ de agua destilada, por medio de un potenciómetro digital, marca Orion, modelo 420, mientras que la acidez total titulable (ATT), fue determinada por titulación con Hidróxido de Sodio $0.1 \mathrm{~N}$, a partir de una muestra de $10 \mathrm{~mL}$ de jugo de agraz.

La transpiración del fruto, se definió por medio de la pérdida gravimétrica en fresco, con respecto al tiempo, tomando muestras de $150 \mathrm{~g}$, como peso inicial, validando los cambios gravimétricos cada 24 h, durante 14 días.

Los datos obtenidos fueron analizados por medio de un análisis de perfiles, usando el paquete "Profile R" de la herramienta software
R v. 3.6.1. En el análisis de perfiles fueron probadas tres hipótesis, paralelismo, coincidencia y horizontalidad (Desjardins, 2005; Bulut \& Desjardins, 2015). Para paralelismo, se utilizó la prueba de Pillai. Los gráficos, se generaron con el paquete GGPLOT para R versión 3.6.

\section{RESULTADOS Y DISCUSIÓN}

No se determinó efecto de los factores y combinaciones en los sólidos solubles totales (Figura 3). Los frutos en etapa madura empacados en polipropileno monorienteado mostraron cierta tendencia a incrementar los SST, entre los 0 y 6 DDT, con una disminución leve entre los 6 a 13 DDT. En investigaciones previas, con almacenamiento a $1^{\circ} \mathrm{C}$, en estado maduro (color del fruto morado), se obtuvo mayor concentración de SST, permaneciendo constante, a través del tiempo de almacenamiento (Rincón Soledad et al. 2012), resultado que coincide como se indica en estudios realizados en pulpa almacenada en condiciones de frío (FrancoTobón et al. 2016).

En general, los tratamientos T1, T3 y T4 sugieren una pérdida continua de SST al final del periodo evaluado, durante el almacenamiento refrigerado, comparado con los SST obtenidos a los 0 DDT.

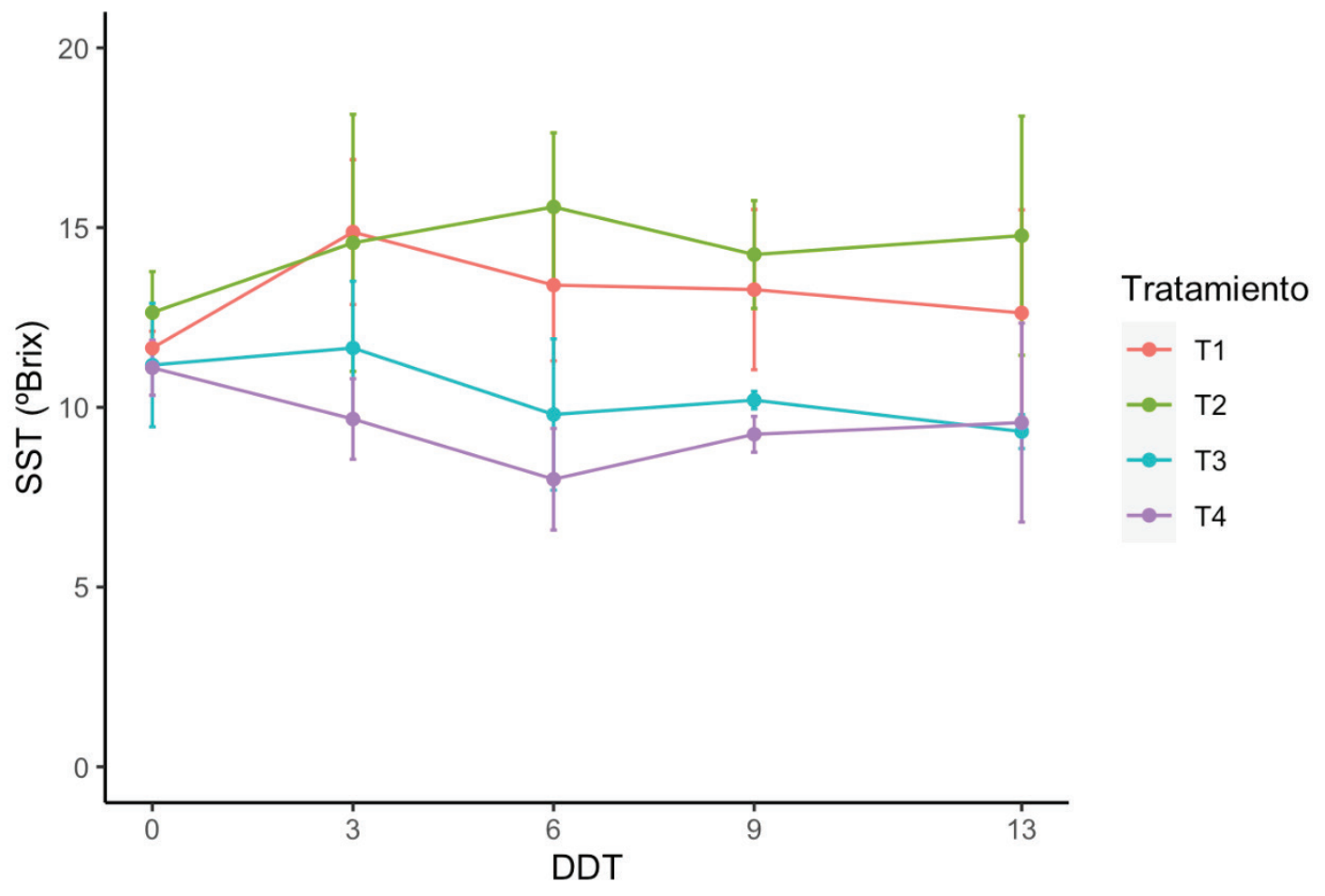

Figura 3. Variación de Sólidos Solubles Totales (SST) del fruto agraz, almacenado a $2^{\circ} \mathrm{C}$ y $90 \% \mathrm{HR}$, durante 12 días, con frecuencia cada 3 días, para 4 tratamientos.

*Paralelismo (p-valor 0,3027), coincidencia ( $\operatorname{Pr}>$ F 9,02 e-6), Horizontalidad ( $\mathrm{p}$-valor 0,5911). T1= morado x polietileno biorientado; T2=morado x Polipropileno Monorientado; T3=rojo x polietileno biorientado y T4= rojo x Polipropileno Monorientado. Las barras de error corresponden a la desviación estándar. 
La reducción de SST en los tratamientos T3 y T4, en los instantes 0 a 3 DDT, se asocia con un gasto de energía, como consecuencia de la respiración del fruto, lo que concuerda con Kays et al. (2004), aunque, en este caso, se prolongó hasta los 3 DDT. De los periodos 6 a 13 DDT, un efecto de concentración, ocasionó una reducción del peso del fruto, fomentando un aumento de los SST, resultado que coincide con lo manifestado por Ávila Rodríguez et al. (2007), siendo particularmente evidente en los tratamientos T2 y T4, para los periodos 9 a 13 DDT.

Por otra parte, la tendencia de aumento de SST del tratamiento T2 es argumentada por la pérdida de humedad creciente, en la medida que acrecienta el periodo de almacenamiento del fruto en comparación con la tasa de desdoblamiento de azúcar ocurrido durante el proceso de respiración (Yao et al. 2018), afectando el metabolismo del fruto y, por lo tanto, sus reservas energéticas.

El pH, se incrementó en todos los tratamientos de 2,9 a 3,1, en el periodo de evaluación (Figura 4). Los frutos en etapa morado empacado en polipropileno monorientado sufrió alteraciones en magnitud de este parámetro, para los 5 instantes evaluados; al final del periodo (13 DDT), alcanzó un $\mathrm{pH}$ similar al presentado al inicio de los tratamientos. Los resultados de variación en el pH, a través del tiempo de almacenamiento, son muy similares a los reportados por Ávila Rodríguez et al. (2007), en almacenamiento a baja temperatura. El tratamiento T2 presentó las menores variaciones en $\mathrm{pH}$, mientras se adelantó la evaluación del fruto de agraz bajo refrigeración, con frutos morados contenido en el empaque de Polipropileno Monorientado (E2).

Para arándanos $V$. corymbusom completamente maduros, se registran valores de $\mathrm{pH}$ entre 2,5 y 3,4 (Saftner et al. 2008), siendo coherente con los resultados alcanzados para agraz. Además, el incremento generalizado de $\mathrm{pH}$ en los tratamientos, en los periodos 0 a 6 DDT, se puede explicar, porque cuando se origina el llenado de frutos, la formación de sustratos, como sacarosa y glucosa, ocurre por cotransporte paralelo (simporte), con una alta participación de iones de hidrógeno $\left(\mathrm{H}^{+}\right)$, concentración de iones que disminuye a nivel vacuolar, mientras ocurren las últimas fases de maduración (Ávila Rodríguez et al. 2007). La disminución de pH, visible en los 4 tratamientos, para los periodos 6 a 13 DDT, supone un incremento de ácidos orgánicos, lo que coincide, además, con el aumento de Acidez Total Titulable (AT'T), para el mismo periodo; esto es, una relación inversamente proporcional entre el pH y la ATT, en esos periodos específicos.

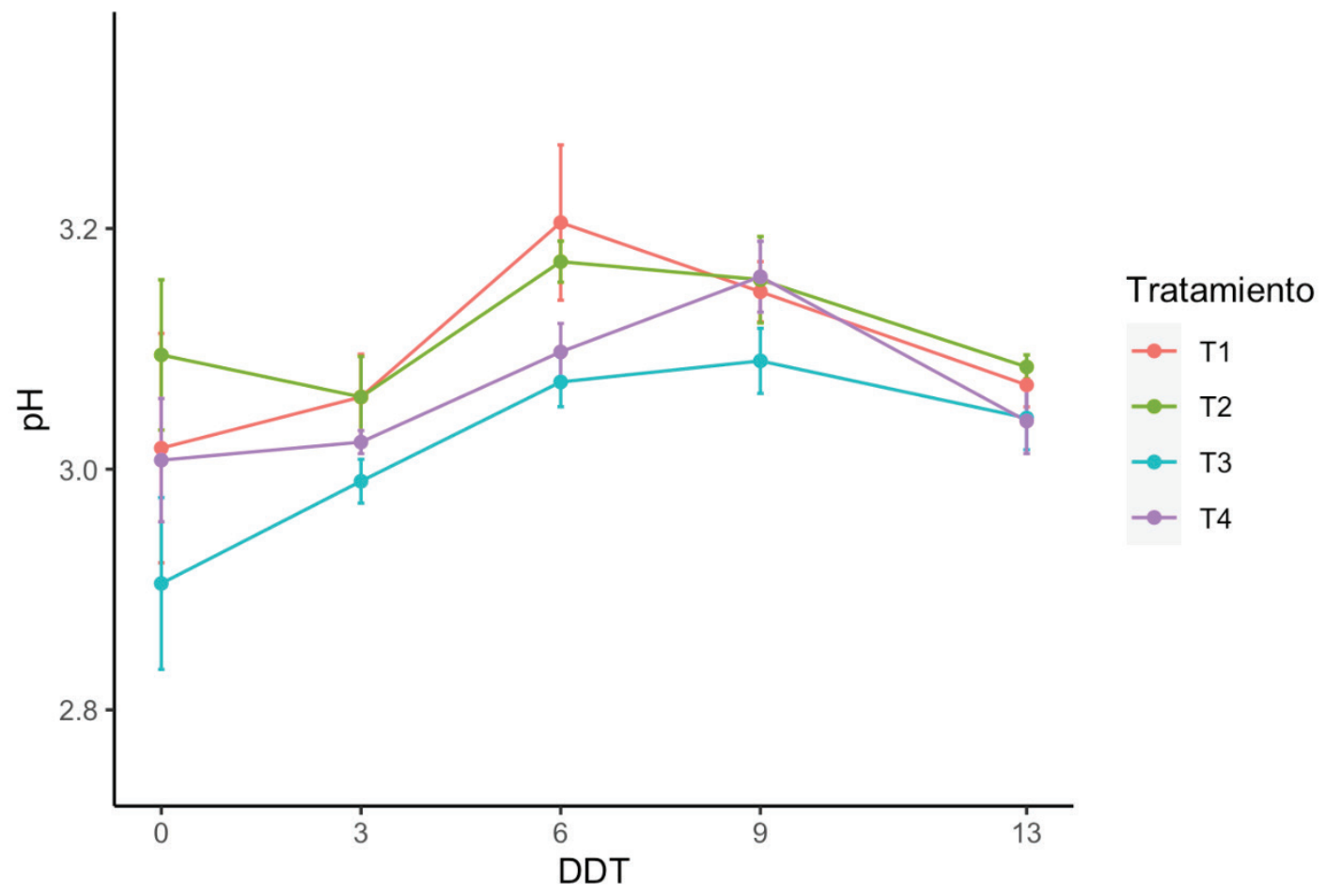

Figura 4. Variaciones de $\mathrm{pH}$ en frutos de agraz, en un periodo de 12 días, para 4 tratamientos.

Paralelismo (p-valor 0,0130). T1 = morado x E1; T2=morado x E2; T3=rojo x E1 y T4= rojo x E2. Las barras de error corresponden a la desviación estándar. 


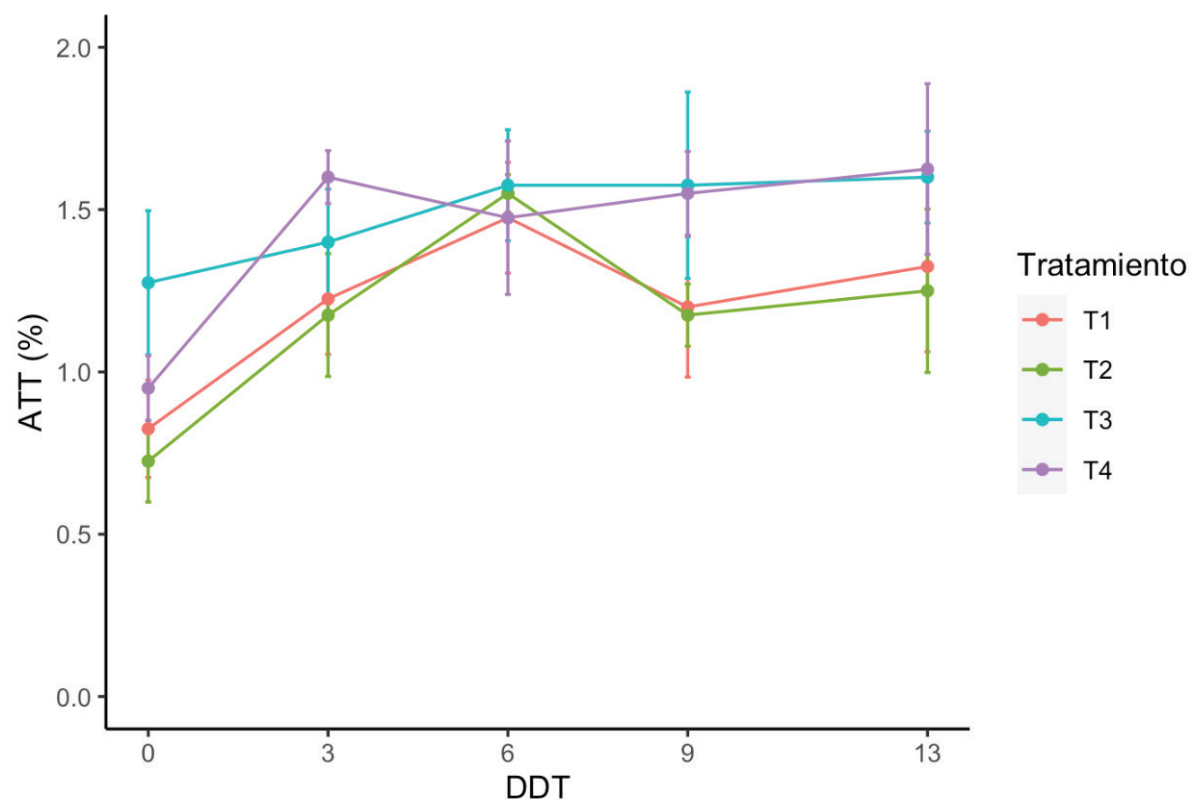

Figura 5. Acidez Total Titulable (ATT) evaluada en agraz, para un periodo total de 12 días, con frecuencia cada 3 días.

*Paralelismo ( $\mathrm{p}$-valor 0,122), coincidencia $(\mathrm{Pf}>\mathrm{f}$ 0,000635), horizontalidad ( $\mathrm{p}$-valor 5,086 e-5). $\mathrm{T} 1=$ morado $\mathrm{x}$ polietileno biorientado; $\mathrm{T} 2=$ morado $\mathrm{x}$ Polipropileno Monorientado; T3=rojo x polietileno biorientado y T4= rojo $\times$ Polipropileno Monorientado. Las barras de error corresponden a la desviación estándar.

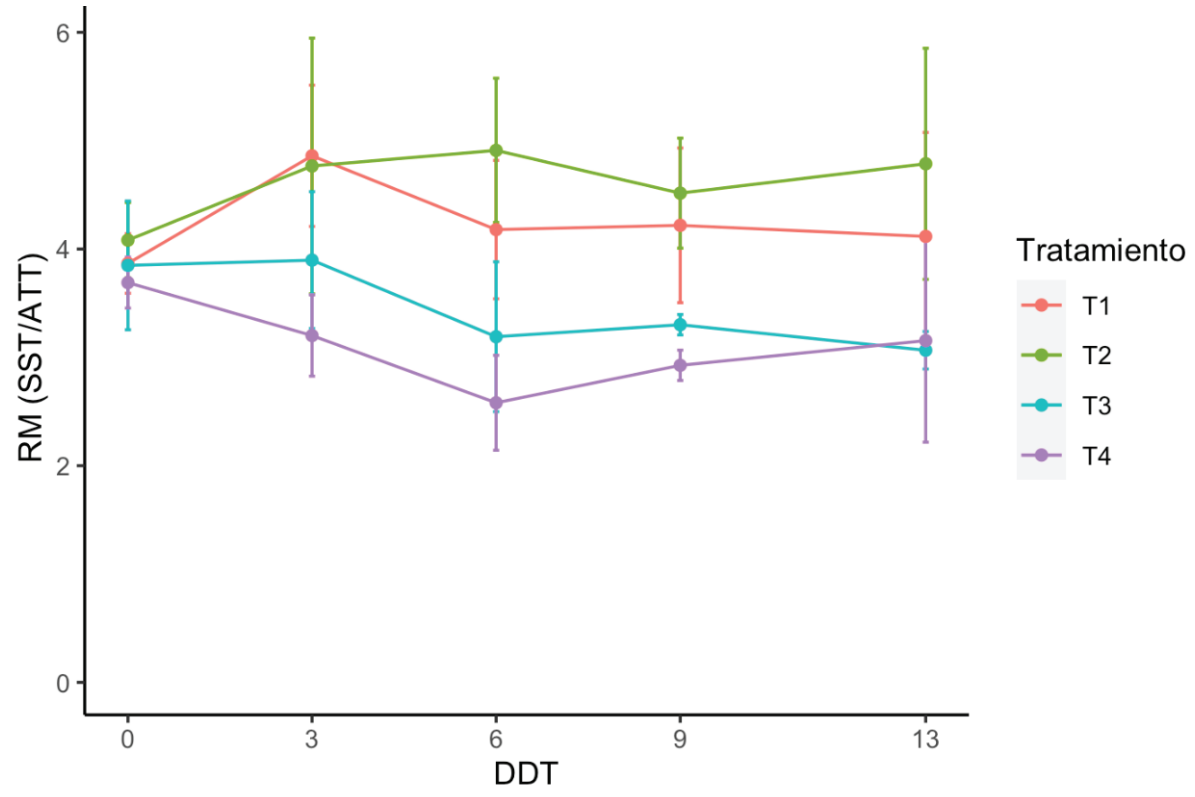

Figura 6. Índice de cosecha obtenido a partir de la relación entre Sólidos Solubles Totales y Acidez Total Titulable (SST/ATT), en un periodo de 12 días, y 4 tratamientos.

*Paralelismo ( $\mathrm{p}$-valor 0,296), coincidencia ( $\operatorname{Pr}>\mathrm{f} 2,31 \mathrm{e}-5)$, horizontalidad ( $\mathrm{p}$-valor 0,2591). $\mathrm{T} 1=$ morado $x$ polietileno biorientado; $\mathrm{T} 2=$ morado x Polipropileno Monorientado; T3=rojo x polietileno biorientado y T4= rojo x Polipropileno Monorientado. Las barras de error corresponden a la desviación estándar. 
La variable ATT presentó variación respecto al tiempo y a los tratamientos (Figura 6). Los tratamientos T1, T2 y T3 presentaron incrementos en los porcentajes de ATT (Figura 5), entre los 0 a 6 DDT, con excepción al tratamiento T4, que evidenció un aumento en todos los tiempos evaluados, sumado a un leve aumento en el tratamiento T3, también para los instantes 6 a 13 DDT, con variaciones generalizadas para los 4 tratamientos, entre $0,8 \%$ y $1,6 \%$. Magnitskiy \& Ligarreto (2009) presentaron resultados en donde ATT, en promedio, alcanzó $1,44 \%$, resultados consistentes con los reportados en el presente estudio, mientras que Ávila Rodríguez et al. (2007) reportaron ATT, en un rango entre $1,44 \%$ a $1,63 \%$, para una evaluación coincidente con frecuencias cada 3 días.

Los tratamientos T1 y T2 demostraron un comportamiento similar, en términos de variación de ATT, con incremento entre los periodos 0 a 6 y 9 a 13 DDT y reducción en el periodo comprendido entre 6 y 9 DDT. La disminución de ATT sugiere que los ácidos orgánicos son utilizados durante el proceso de respiración, con posibilidad de transformarlos en azúcares (Buitrago Guacaneme et al. 2015). Las bajas temperaturas $\left(2^{\circ} \mathrm{C}\right)$ durante el almacenamiento, suponen un desdoblamiento de los azúcares y encogimiento del fruto, como consecuencia de la pérdida de agua, durante el periodo de almacenamiento refrigerado, a pesar de mantener $90 \%$ de humedad relativa (HR).

La relación de madurez establece la relación de Sólidos Solubles Totales (STT), con respecto a la ATT; sin embargo, en el presente estudio, se encontró efecto solo por incidencia de los tratamientos (Figura 6). La relación SST/ATT señala, para los 4 tratamientos, un rango entre 2,5 y 5,0, resultado que coinciden con lo reportado por Buitrago Guacaneme et al. (2015), definiendo la relación SST/ ATT, como un indicador simple de la calidad de la fruta, en donde magnitudes bajas de la relación de madurez, sugieren un fruto con alta capacidad para resistir el ataque de microorganismos durante la poscosecha, con ${ }^{\circ}$ Brix superior a la ATT, demostrando un balance entre azúcares y ácidos, combinando características de aroma, textura y sabor, apetecibles por el consumidor. El balance entre azúcares y ácidos fue evidente en los 4 tratamientos; sin embargo, el tratamiento T2 fue el que presentó menor variación de la relación de madurez, cuando se dispone de un fruto morado, almacenado en el empaque de Polipropileno Monorientado (E2), por un periodo de 12 días.

En la presente investigación, se puede concluir que el fruto de agraz clasificado como rojo no modifica sus características internas, independientemente del material del empaque dispuesto para el almacenamiento refrigerado; sin embargo, el empaque de polipropileno monoorientado, genera un efecto favorable en la acumulación sólidos solubles totales en frutos categorizados como morados, condición necesaria para preservar las características de sabor del agraz. En general, el fruto de agraz tiende a modificar sus características internas durante el periodo postcosecha, característico de frutos climaterios (Rincón Soledad et al. 2012).

Las características físico químicas de un fruto de agraz, clasificado como morado, son altamente susceptibles a los cambios de las condiciones de almacenamiento. Adicionalmente, definir el material de los empaques es fundamental para preservar el mayor tiempo posible los parámetros de calidad del fruto, de acuerdo con las exigencias del consumidor.

Conflicto de intereses: El trabajo fue preparado y revisado por todos los autores, no existe ningún conflicto de intereses que pongan en riesgo la validez de los resultados. Financiación: La investigación fue financiada por la Universidad de Ciencias Aplicadas y Ambientales.

\section{REFERENCIAS}

1. ALMENAR, E.; SAMSUDIN, H.; AURAS, R.; HARTE, B.; RUBINO, M. 2008. Postharvest shelf life extension of blueberries using a biodegradable package. Food Chem. 110(1):120-127. https://doi.org/10.1016/j.foodchem.2008.01.066

2. ALZATE-ARBELÁEZ, A.F.; DORTA, E.; LÓPEZALARCÓN, C.; CORTÉS, F.B.; ROJANO, B.A. 2019. Immobilization of Andean berry (Vaccinium meridionale) polyphenols on nanocellulose isolated from banana residues: A natural food additive with antioxidant properties. Food Chem. 294:503-517. https://doi.org/10.1016/j.foodchem.2019.05.085

3. ÁVILA RODRÍGUEZ, H.G.; CUSPOCA RIVEROS, J.A.; FISCHER, G.; LIGARRETO MORENO, G.A.; QUICAZÁN DE CUENCA, M.C. 2007. Caracterización fisicoquímica y organoléptica del fruto de agraz (Vaccinium meridionale Swartz) almacenado a $2^{\circ} \mathrm{C}$. Rev. Fac. Nac. Agron. Med. 60(2):4179-4193.

4. BRICEÑO, B.; MORILLO, G. 2002. Catálogo abreviado de las plantas con flores de los páramos de Venezuela. Parte I. Dicotiledóneas (Magnoliopsida). Acta Botánica Venez. 25(1):1-46.

5. BUITRAGO GUACANEME, C.M.; RINCÓN SOLEDAD, M.C.; BALAGUERA LÓPEZ, H.E.; LIGARRETO MORENO, G.A. 2015. Tipificación de Diferentes Estados de Madurez del Fruto de Agraz (Vaccinium meridionale Swartz). Rev. Fac. Nac. Agron. Med. 68(1):7521-7531. https://doi.org/10.15446/rfnam.v68n1.47840

6. BULUT, O.; DESJARDINS, C.D. 2015. Package 'profileR' (No. 0.3). Disponible desde Internet en: https://cran.rproject.org/web/packages/profileR/ profileR.pdf (con acceso 10/05/2020).

7. DESJARDINS, C.D. 2005. Journal of Statistical Software Profile Analysis of Multivariate Data in R: An Introduction to the profileR Package. J. Stat. Soft. 55(2):1-29.

8. DIAZ-URIBE, C.; VALLEJO, W.; CAMARGO, G.; MUÑOZACEVEDO, A.; QUIÑONES, C.; SCHOTT, E. 2019. 
Chemistry Potential use of an anthocyanin-rich extract from berries of Vaccinium meridionale Swartz as sensitizer for TiO 2 thin films - An experimental and theoretical study. J. Photo. Photobiology. A: Chemistry. 384:112050. https://doi.org/10.1016/j.jphotochem.2019.112050

9. FRANCO-TOBÓN, Y.N.; ROJANO, B.; ALZATEARBELÁEZ，A.F.; RESTREPO-FLOREZ，C.E.; RIVERO-BARRIOS, D.M.; MALDONADO-CELIS, M.E. 2016. Efecto del tiempo de almacenamiento sobre propiedades fisicoquímicas y antioxidantes de productos derivados del fruto agraz (Vaccinium meridionale Swartz). Vitae. 23:184-193.

http://dx.doi.org/10.17533/udea.vitae.v23n3a04

10. GARZÓN, G.A.; NARVÁEZ, C.E.; RIEDL, K.M.; SCHWARTZ, S.J. 2010. Chemical composition, anthocyanins, non-anthocyanin phenolics and antioxidant activity of wild blueberry (Vaccinium meridionale Swartz) from Colombia. Food Chem. 122(4):980-986. https://doi.org/10.1016/j.foodchem.2010.03.017

11. GONZÁLEZ, M.; SAMUDIO, I.; SEQUEDA-CASTAÑEDA, L.G.; CELIS, C.; IGLESIAS, J.; MORALES, L. 2017. Cytotoxic and antioxidant capacity of extracts from Vaccinium meridionale Swartz (Ericaceae) in transformed leukemic cell lines. J. Appl. Phar. Sci. 7(3):24-30. https://doi.org/10.7324/JAPS.2017.70305

12. KARACAY, E.; AYHAN, Z. 2010. Physiological, physical, chemical characteristics and sensory evaluation of minimally processed grapefruit segments packaged under modified atmosphere. J. Agric Sci. 16:129-130.

13. KAYS, S.J.; PAULL, R.E.; MOHAMMED, M.; BENÍTEZ, C.E.; CASTRO, H.R.; RICCA, A.P.; MAU, R.F.L. 2004. Postharvest biology. Ed. IICA (USA). 568p.

14. LIGARRETO MORENO, G.; PEREA DALLOS, M.; LARA, A.M.; MUÑOZ, J.D.; MATALLANA, L. 2010. Vaccinium sp -Agraz. En: Perea Dallos, M.; Matallana R., L.P.; Tirado Perea, A. (Eds.), Biotecnología aplicada al mejoramiento de los cultivos de frutas tropicales. Ed Universidad Nacional de Colombia (Bogotá). p.203-232.

15. MAGNiTSKIY, S.; LIGARRETO, G.A. 2009. Plantas de agraz o mortiño (Vaccinium meridionale Swartz): potencial de propagación sexual. En: Ligarreto, G.A. (ed). Perspectivas del cultivo de agraz o mortiño (Vaccinium meridionales Swartz) en la zona altoandina de Colombia. Ed. Universidad Nacional de Colombia (Bogota). p.75-91.

16. MANGARAJ, S.; GOSWAMI, T.K.; MAHAJAN, P.V. 2009. Applications of Plastic Films for Modified Atmosphere
Packaging of Fruits and Vegetables: A Review. Food Eng. Rev. 1(2):133.

https://doi.org/10.1007/s12393-009-9007-3

17. MEDINA CANO, C.I.; MARTÍNEZ BUSTAMANTE, E.; LÓPEZ OROZCO, C.A. 2019. Phenological scale for the mortiño or agraz (Vaccinium meridionale Swartz) in the high Colombian Andean area. Rev. Fac. Nac. Agr. Med. 72(3):8897-8908.

https://doi.org/10.15446/rfnam.v72n3.74460

18. PATIÑO, M.D.P.; LIGARRETO, G.A. 2006. Caracterización morfológica in situ de poblaciones espontáneas de Vaccinium sp. en los departamentos de Boyacá, Cundinamarca y Nariño. Memorias 1er Congreso Colombiano de Horticultura. Soc. Col. Cien. Hort. Bogotá. p.112.

19. RINCÓN SOLEDAD, M.C.; BUITRAGO GUACANEME, C.M.; LIGARRETO MORENO, G.A.; TORRES APONTE, W.S.; BALAGUERA LÓPEZ, H.E. 2012. Comportamiento del fruto de agraz (Vacinium meridionale Swartz) cosechado en diferentes estados de madurez y almacenado en refrigeración. Rev. Fac. Nac. Agro. 65(2):6615-6625.

20. SAFTNER, R.; POLASHOCK, J.; EHLENFELDT, M.; VINYARD, B. 2008. Instrumental and sensory quality characteristics of blueberry fruit from twelve cultivars. Post. Biol. Tech. 49(1):19-26.

https://doi.org/10.1016/j.postharvbio.2008.01.008

21. SOMBOONKAEW, N.; TERRY, L.A. 2010. Physiological and biochemical profiles of imported litchi fruit under modified atmosphere packaging. Post. Biol. Tech. 56(3):246-253. https://doi.org/10.1016/j.postharvbio.2010.01.009

22. VAN WILLIGE, R.W.G.; LINSSEN, J.P.H.; MEINDERS, M.B.J.; VAN DER STEGE, H.J.; VORAGEN, A.G.J. 2002. Influence of flavour absorption on oxygen permeation through LDPE, PP, PC and PET plastics food packaging. Food Add. Cont. 19(3):303-313. https://doi.org/10.1080/02652030110081146

23. VIJAYANAND, P.; YADAV, A.R.; BALASUBRAMANYAM, N.; NARASIMHAM, P. 2000. Storage Stability of Guava Fruit Bar Prepared Using a New Process. LWT - Food. Sci. Tech. 33(2):132-137. https://doi.org/10.1006/fstl.1999.0627

24. WILLS, R.B.; GOLDING J.B. 2016. Postharvest: an introduction to the physiology and handling of fruit and vegetables. Ed. CABI (Wallingford). 174p.

25. YAO, S.; CAO, Q.; XIE, J.; DENG, L.; ZENG, K. 2018. Alteration of sugar and organic acid metabolism in 
postharvest granulation of Ponkan fruit revealed by transcriptome profiling. Post. Bio. Tech. 139:2-11.

https://doi.org/10.1016/j.postharvbio.2018.01.003 